\title{
CHARACTERIZATION OF MAGNETIC NANOPARTICLE-EMBEDDED SU8 FOR MICROACTUATION
}

\author{
K.L. Tsai, M. Ziaei-Moayyed, N. Klejwa, R.N. Candler, W. Hu, S.X. Wang, and R.T. Howe \\ Stanford University, USA
}

\begin{abstract}
SU8 polymer with embedded magnetic nickel nanoparticles (SU8-Ni) can be used as a structural material for microsystems and has the potential to be used for biomedical applications where remote actuation is needed. A composition of SU8-Ni $(12.5 \% \mathrm{Ni}$ nanoparticles and $87.5 \%$ SU8-2010 by weight) is investigated in this paper. Polysilicon torsional actuators were fabricated to measure the saturation magnetic moment of the material, and measurements were validated with alternating gradient magnetometry (AGM). Optical transmittance of the magnetic polymer was measured with a spectrophotometer and compared to standard SU8-2010.
\end{abstract}

\section{INTRODUCTION}

SU8 is an epoxy based negative photoresist that has several advantages as a micromechanical system material, including biocompatibility [1], chemical resistance, thermal stability, low cost, and mechanical compliance for large deflections [2]. Using primarily SU8, Hill et al. [3] created a Fabry-Perot pressure sensor for blood pressure measurements. Chen et al. [4] used SU8 on silicon cantilevers to create underwater shear sensors. Magnetized SU8 could combine the advantages of SU8 for biomedical applications (specifically biocompatibility and chemical resistance) with the possibility of long-distance non-invasive magnetic actuation. Previous work [5] demonstrated the feasibility of magnetically deflecting cantilevers made from SU8-Ni but did not fully characterize the magnetic properties of the material. A full understanding of the material properties of nanoparticle embedded polymers is necessary if they are to be successfully used in vivo. In this work, the magnetic and optical properties (e.g., saturation magnetic moment and transmittance) for one nickel concentration of this material are measured, which is a critical starting point for understanding its capabilities for use in microsystems.

\section{MAGNETIC CHARACTERIZATION}

A magnetic MEMS actuator previously developed for micromirror applications consisted of electroplated nickel on a polysilicon plate supported by a pair of torsional beams $[6,7]$. Its torsional design allowed for large angular displacement under moderate magnetic forces.

A similar device design is used here as a platform for magnetic characterization of the SU8-Ni material (Figure 1). The large deflections give a large sensitivity to force, which enables finer resolution measurements of magnetic properties of the material on the device.

\section{Fabrication Process}

The SU8-Ni was prepared by mixing SU8-2010 photoresist (MicroChem, MA, USA), 87.5\% by weight, and nickel powder (100 nm diameter, Argonide, FL, USA), $12.5 \%$ by weight, with a rotisserie device for at least 12 hours to achieve a uniform suspension. Figure 2 shows the fabrication process for the torsional actuator used to characterize the magnetic properties of the SU8-Ni material.

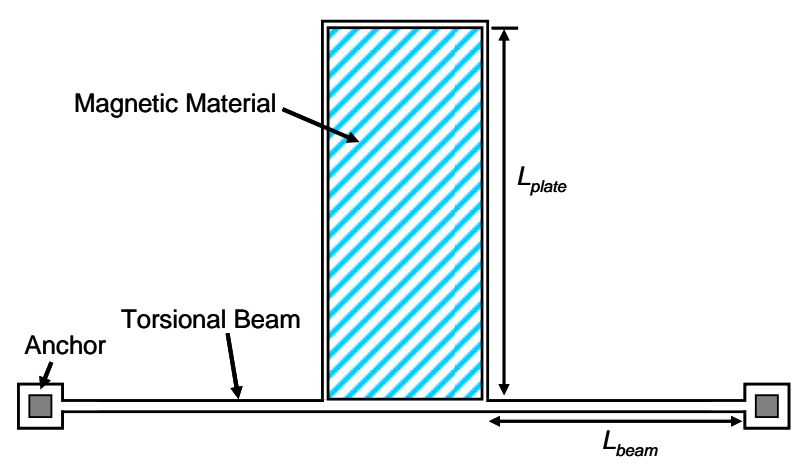

Figure 1. Top view of the torsional magnetic actuator used to characterize the SU8-Ni. $L_{\text {plate }}=430 \mu \mathrm{m}$ and $L_{\text {beam }}=400 \mu \mathrm{m}$ (figure not to scale).

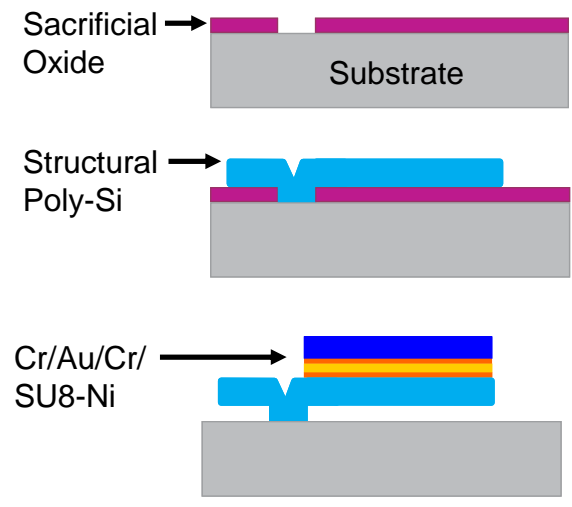

Figure 2. Fabrication process. (a) Deposit and pattern sacrificial oxide. (b) Deposit and pattern polysilicon structure layer. (c) Deposit and pattern $\mathrm{Cr} / \mathrm{Au} / \mathrm{Cr} / \mathrm{SU} 8-\mathrm{Ni}$. Release device with $\mathrm{HF}$ oxide etch and CPD.

A $1 \mu \mathrm{m}$ thick sacrificial oxide layer was first deposited on a $<100>$ silicon substrate at $400{ }^{\circ} \mathrm{C}$ by low pressure chemical vapor deposition (LPCVD) and patterned via plasma etching. A $1.4 \mu \mathrm{m}$ thick structural polysilicon layer was then deposited by LPCVD and patterned via plasma etching. Next, a $10 \mathrm{~nm} \mathrm{Cr/100} \mathrm{nm} \mathrm{Au/10}$ $\mathrm{nm} \mathrm{Cr}$ metal stack was deposited via sputtering. This metal stack was necessary for proper adhesion of the SU8-Ni during device release. A $15 \mu \mathrm{m}$ thick layer of the SU8-Ni was then spin-coated and patterned according to standard processing procedures for SU8-2010. The spin speed used for the SU8-Ni was the same as that for standard SU8-2010 (e.g., $1625 \mathrm{rpm}$ for $15 \mu \mathrm{m}$ thickness). Scanning electron micrograph (SEM) images were taken to measure the thickness of the SU8-Ni and confirmed that the SU8 viscosity was not affected by the addition of the nanoparticles. To prevent the SU8-Ni layer from cracking, extra care was taken to avoid sudden temperature changes during the baking steps. Finally, devices were released in 6:1 buffered HF and dried using critical point drying to prevent stiction (Figure 3). 


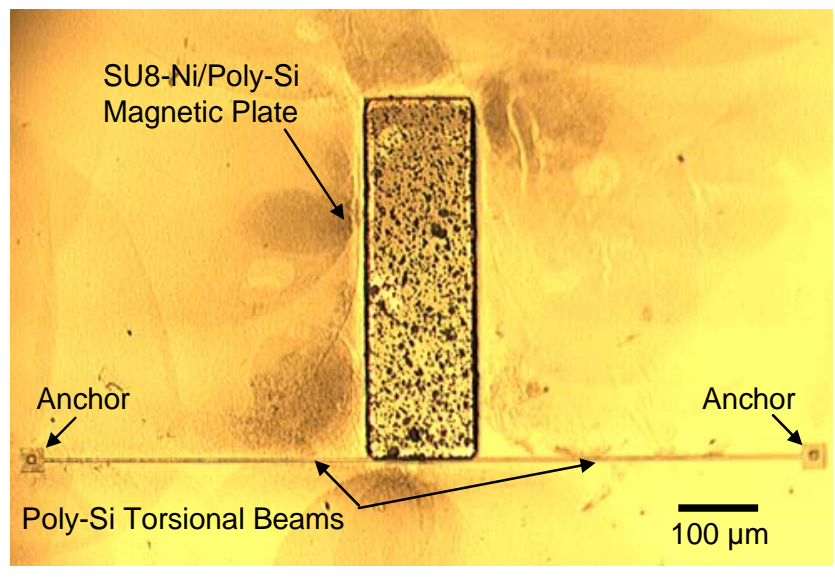

Figure 3. Optical micrograph of magnetic actuator composed of a SU8-Ni/Poly-Si plate (SU8-Ni: $430 \mu \mathrm{m}$ (l) $\times 130 \mu \mathrm{m}$ (w) $\times 15 \mu \mathrm{m}$ (t)) suspended by a pair of poly-Si torsional beams (400 $\mu \mathrm{m}$ (l) $x$ $3.2 \mu \mathrm{m}(w) \times 1.4 \mu \mathrm{m}(\mathrm{t}))$.

\section{Experimental Results}

Devices were tested using a permanent magnet mounted onto a micropositioner for precision position control and a probe station for rough position control (Figure 4). The actuator chip was mounted vertically under the microscope for optical measurement of deflection. The magnet position was controlled by the micropositioner.

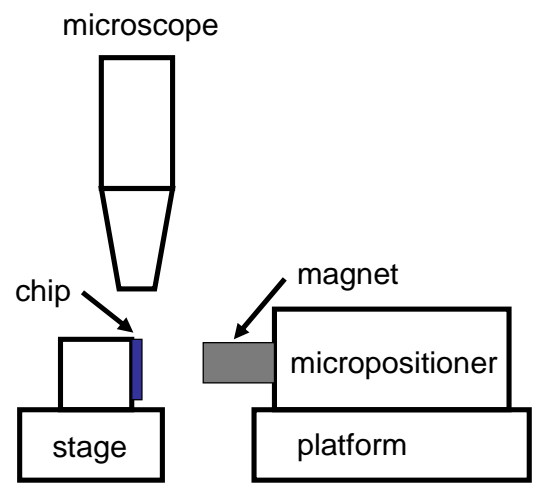

Figure 4. Schematic of probe station test setup for measuring actuator deflection vs. magnet distance (not to scale). The chip holder is non-magnetic.
A Hall probe was used to measure the magnetic field strength as a function of distance from the magnet surface (Figure 5).

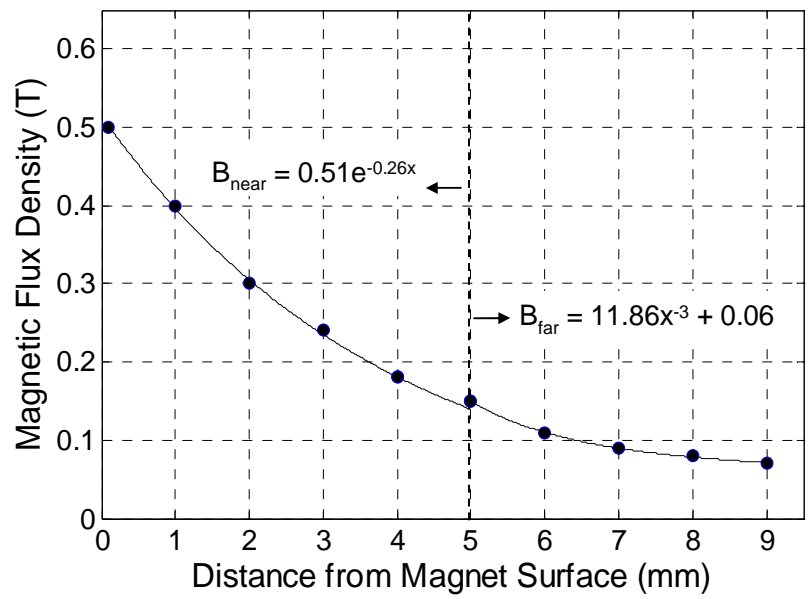

Figure 5. Calibration experiment results showing magnetic flux density (B) as a function of distance from magnet surface ( $x$ ). Near field approximation (i.e., $x<5 \mathrm{~mm}$ ) shows exponential relationship between $B$ and $x$, while far-field approximation (i.e., $x$ $>5 \mathrm{~mm}$ ) shows $1 / x^{3}$ relationship between $B$ and $x$.

A magnetic actuator composed of $12.5 \% \mathrm{Ni}$ nanoparticles (100 nm diameter) and 87.5\% SU8-2010 by weight was tested. Deflection of the actuator was observed when the magnet was brought into close proximity to the actuator (Figure 6).

Figure 7a shows the bending angle and vertical displacement as a function of magnet distance. The data obtained from the calibration experiment (Figure 5) was used to convert magnet distance to magnetic field strength (Figure $7 \mathrm{~b}$ ), assuming the magnetic actuator has a negligible effect on the magnetic field.

Quantitative measurements of the bending angle $(\theta)$ were used to determine the vertical displacement $(d)$ at the free end of the magnetic plate using the relation:

$$
d=\sin (\theta) L_{\text {plate }}
$$

where $L_{\text {plate }}$ is the length of the magnetic plate $(430 \mu \mathrm{m})$.
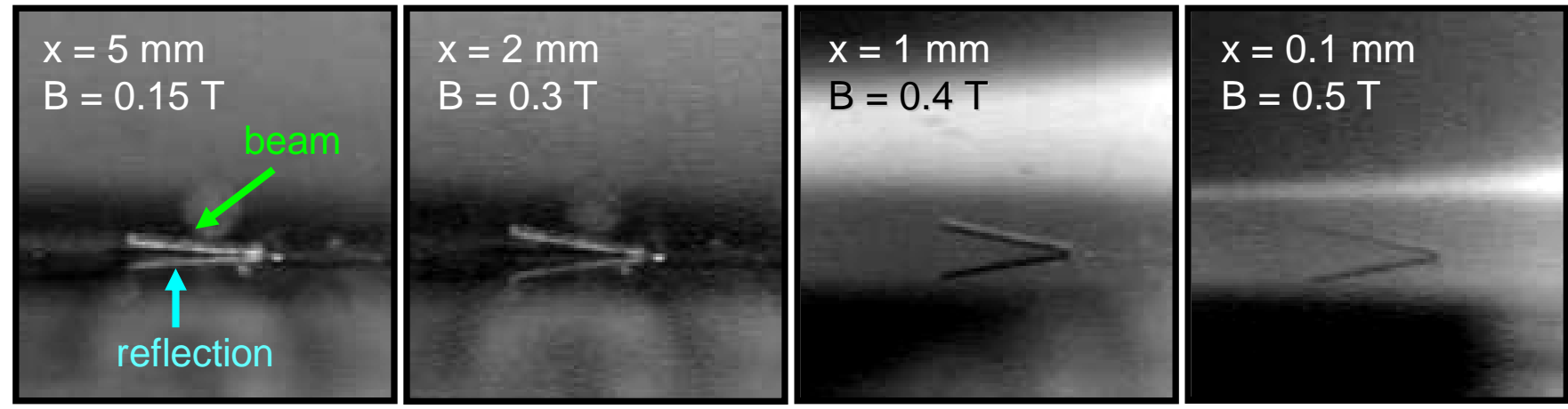

Figure 6. (Side view) Deflection of the magnetic actuator with decreasing magnet distance (x) and increasing magnetic flux density (B). 
(a)

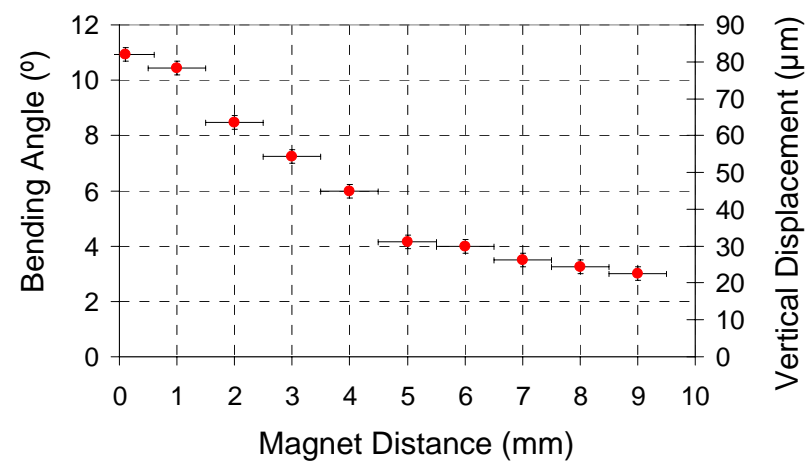

(b)

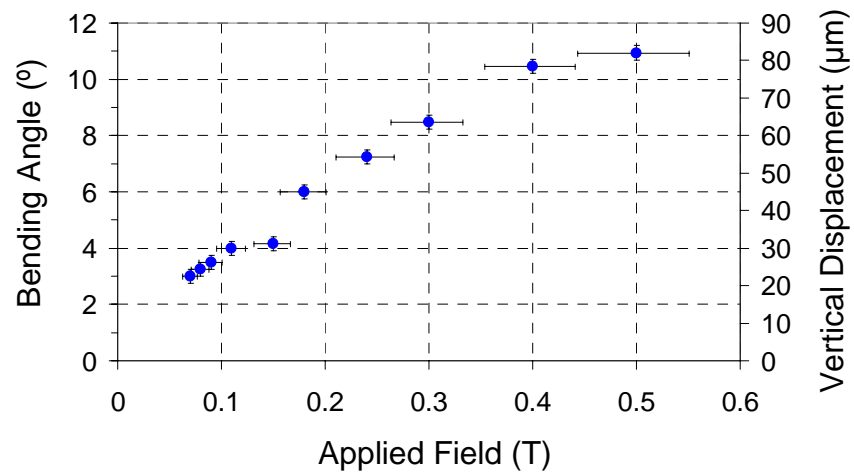

Figure 7. Bending angle and vertical deflection of magnetic actuator (a) as a function of magnet distance and (b) as a function of applied magnetic field.

The bending angle was also used to calculate the torque $(T)$ exerted on the magnetic plate using the general expression for torsional deformation of a rectangular beam (Figure 8):

$$
T=\frac{2 K G}{L_{\text {beam }}} \theta
$$

where $L_{\text {beam }}$ is the length of a single torsional beam (Figure 1 ) and $G=E /[2(1+v)]$ is the shear modulus of elasticity. For polysilicon, $E=169 \mathrm{GPa}$ [8] and $v=0.22$, and the shear modulus was found to be $69.3 \mathrm{GPa}$. $K$ is the polar moment of inertia for a torsional bar with rectangular cross section [9, 10]. For a torsional bar with width and thickness of $2 a$ and $2 b$, respectively, it is given by the equation:

$$
K=a b^{3}\left[\frac{16}{3}-3.36 \frac{b}{a}\left(1-\frac{b^{4}}{12 a^{4}}\right)\right] \quad \text { for } a \geq b
$$

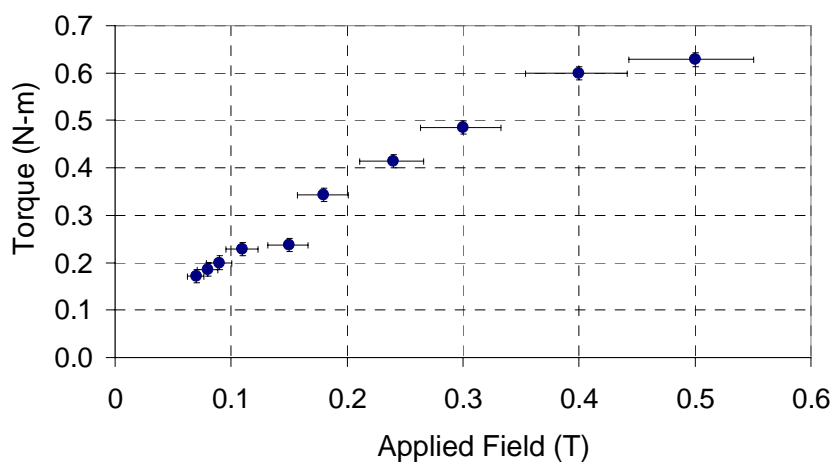

Figure 8. Torque vs. applied magnetic field.

A basic model was developed to calculate the magnetic moment of the SU8-Ni from the experimentally-derived torque on the magnetic plate. Because the magnetic nanoparticles are suspended uniformly throughout the plate, the torque can be approximated as a function of the average magnetic moment $\left(\mathrm{m}_{\mathrm{avg}}\right)$, assumed to be at the center of the plate, and the field gradient at the center of the plate:

$$
T=F_{\text {mag }} \frac{L_{\text {plate }}}{2}=\nabla\left(m_{\text {avg }} \cdot B\right) \frac{L_{\text {plate }}}{2}
$$

where $m$ = magnetic moment and $B=$ magnetic flux density.

In saturation, the magnetization is constant, so $\nabla m_{\text {avg }}=0$. Therefore, (4) can be simplified as:

$$
T=\left(m_{s, a v g} \cdot \nabla B\right) \frac{L_{\text {plate }}}{2}
$$

and

$$
m_{s, a v g}=\frac{2 T}{(\nabla B) L_{\text {plate }}}
$$

where $m_{s, a v g}$ is the average magnetic moment in saturation and $\nabla B$ is the field gradient at the center of the plate. Alternating gradient magnetometry (AGM) measurements (Figure 9) suggest the SU8$\mathrm{Ni}$ is in saturation for $B>0.2 \mathrm{~T}$, so this was the range of data used to calculate $m_{s, a v g}$. Substituting $\nabla B$ from the calibration experiment (Figure 5 ) and $T$ from the deflection experiment (Equation 2) into (6), the average saturation magnetic moment, of the SU8-Ni was found to be $6.4 \mathrm{nA}-\mathrm{m}^{2}$.

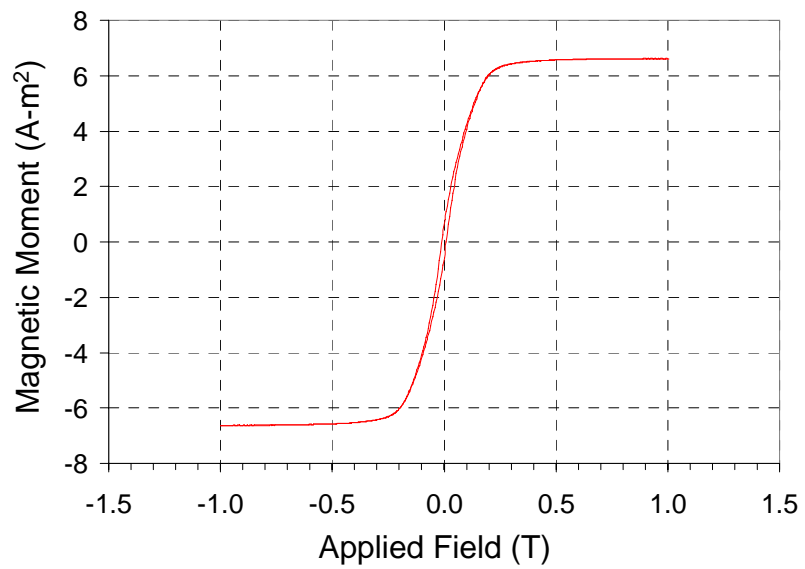

Figure 9. Alternating gradient magnetometry (AGM) results showing magnetic moment vs. applied magnetic field. Measurement shows narrow hysteresis and small coercivity. 
The AGM measurements confirmed the validity of the experimentally-derived value for $\mathrm{m}_{\mathrm{s}, \mathrm{avg}}$ and provided further insight into the magnetic properties of SU8-Ni (Figure 9). Specifically, its small coercivity and narrow hysteresis verified that the ferromagnetic property of the Ni nanoparticles was not affected by the SU8. The measurements and their interpretation using the first-order analytical model were consistent with the saturation magnetic moments expected from this class of nanoparticles combined with their density in SU8 (Table 1).

Table 1. Comparison of expected and measured $m_{s}$ values.

\begin{tabular}{|c|c|}
\hline Saturation Magnetic Moment $\left(\mathrm{m}_{\mathrm{s}}\right)$ & Value \\
\hline $\begin{array}{c}\text { Expected } \\
\text { (based on } 12.5 \% \text { wt Ni concentration) }\end{array}$ & $\begin{array}{llr}6.8 & \mathrm{nA}^{2} \mathrm{~m}^{2}(\mathrm{SI}) \\
& \mu \mathrm{emu}(\mathrm{CGS}) \\
\end{array}$ \\
\hline $\begin{array}{c}\text { Measured } \\
\text { (based on AGM results (Fig 9)) }\end{array}$ & $\begin{array}{llr}6.6 & \mathrm{nA}^{2} \mathrm{~m}^{2} & \text { (SI) } \\
& \mu \mathrm{emu} & \text { (CGS) }\end{array}$ \\
\hline $\begin{array}{c}\text { Measured } \\
\text { (based on deflection experiment (Eq 6)) }\end{array}$ & $\begin{array}{ll}6.4 & \text { nA-m }^{2}(\mathrm{SI}) \\
& \mu \mathrm{emu}(\mathrm{CGS})\end{array}$ \\
\hline
\end{tabular}

\section{OPTICAL CHARACTERIZATION}

A 4" quartz substrate was spin-coated with a $15 \mu \mathrm{m}$ thick layer of SU8-Ni (87.5\% SU8-2010 and $12.5 \% \mathrm{Ni}$ by weight) for characterizing the optical properties of the magnetic polymer. The SU8-Ni film was imaged and cured following standard processing guidelines for SU8-2010 (e.g. pre-baking, exposing, post-exposure baking, developing, and hard-baking). The transmittance spectrum of the SU8-Ni film was then measured using a spectrophotometer (Figure 10).

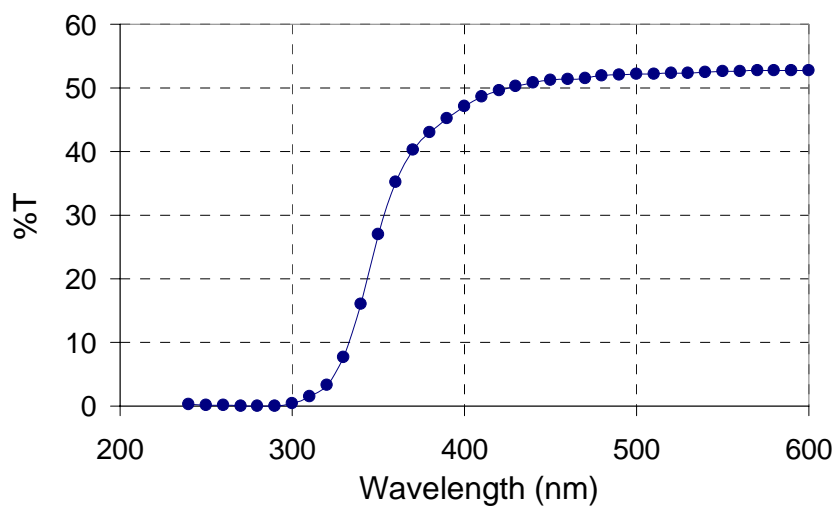

Figure 10. Transmittance of SU8-Ni (12.5\% Ni by weight).

Compared to standard SU8-2010, the SU8-Ni composite showed lower transmittance in the $350-600 \mathrm{~nm}$ range (53\% vs. 91\%), but the transition between absorption and transmission (i.e. at $350 \mathrm{~nm}$ ) was almost unaffected. This was to be expected since the diameter of the nanoparticles $(100 \mathrm{~nm})$ is well below the wavelength of the transmitted light. Assuming the SU8-Ni behaves as a dispersive medium, the transmittance can be formulated according to the Beer-Lambert law:

$$
T=e^{-\left(\alpha_{S U 8}+\alpha_{N i}\right) x}
$$

where $\alpha_{\text {SU8 }}$ is the attenuation coefficient of the SU8, $\alpha_{\mathrm{Ni}}$ is the attenuation coefficient of the Ni particles, and $x=$ path length (i.e., film thickness). Using the transmittance data at a particular wavelength, the attenuation coefficients for pure SU8-2010 and the SU8-Ni (12.5\% Ni by weight) were determined. For $\lambda=500 \mathrm{~nm}$,
$\mathrm{T}_{\mathrm{SU} 8}=0.91$ and $\mathrm{T}_{\mathrm{SU8}-\mathrm{Ni}}=0.53$. Therefore, $\alpha_{\mathrm{SU} 8}=62.87 \mathrm{~cm}^{-1}$ and $\alpha_{\text {SU8-Ni }}=423.25 \mathrm{~cm}^{-1}$. This measurement is important for determining the maximum concentration of $\mathrm{Ni}$ allowable in a sample with a minimum required transmittance.

\section{CONCLUSION}

Magnetic SU8 holds promise as an enabling material for novel sensors and actuators. The saturation magnetic moment of a SU8-Ni composite was measured with a torsional actuator platform and was within $6 \%$ of the expected value. The optical transmittance of the material was also measured. Future work includes analyzing different concentrations of $\mathrm{Ni}$ nanoparticles as well as investigating other magnetic and polymer materials, which will allow the engineering of novel composite or polymer-only microactuators with optimized magnetic and optical properties.

\section{ACKNOWLEDGEMENTS}

Fabrication work was performed at the Stanford Nanofabrication Facility (a member of the National Nanotechnology Infrastructure Network) supported by the NSF under Grant ECS-9731293, its lab members, and the industrial members of the Stanford Center for Integrated Systems. This work was supported by an NSF Graduate Research Fellowship. The authors would like to thank J Provine for helpful discussions.

\section{REFERENCES}

[1] N. Chronis and L. P. Lee, "Electrothermally Activated SU-8 Microgripper for Single Cell Manipulation in Solution", Journal of Microelectromechanical Systems, 14, 4 (2005).

[2] H. Lorenz, M. Despont, N. Fahmi, N. LaBianca, P. Renaud, and P. Vettiger, "SU-8: A Low-cost Negative Resist for MEMS”, Journal of Micromechanics and Microengineering, 7 (1997).

[3] G.C. Hill, R. Melamud, F.E. Declercq, A.A. Davenport, I.H. Chan, P.G. Hartwell, and B.L. Pruitt, "SU-8 MEMS FabryPerot Pressure Sensor", Sensors and Actuators A: Physical, 138, 1 (2007).

[4] N. Chen, J. Chen, J. Engel, S. Pandya, C. Tucker and C. Liu, "Development and Characterization of High Sensitivity Bioinspired Artificial Haircell Sensor”, The $12^{\text {th }}$ Solid State Sensors, Actuator, and Microsystems Workshop (Hilton Head 2006), Hilton Head Island, SC, 6/4-8/06.

[5] N. Damean, B. A. Parviz, J. N. Lee, T. Odom, and G. M. Whitesides, "Composite Ferromagnetic Photoresist for the Fabrication of Microelectromechanical Systems", Journal of Micromechanics and Microengineering, 15 (2005).

[6] J. W. Judy and R. S. Muller, "Magnetic Microactuation of Torsional Polysilicon Structures", $8^{\text {th }}$ International Conference on Solid-State Sensors and Actuators Digest of Technical Papers (Transducers 1995), Stockholm, Sweden, 6/25-29/95, vol. 1, pp. 332-335.

[7] J. W. Judy and R. S. Muller, "Magnetically Actuated, Addressable Microstructures", Journal of Microelectromechanical Systems, 6, 3 (1997).

[8] W. C. Young and R. G. Budynas, Roark's Formulas for Stress and Strain, $7^{\text {th }}$ Edition, McGraw-Hill (2002).

[9] C. Liu, Foundations of MEMS, Upper Saddle River, NJ, Pearson/Prentice Hall (2006).

[10] W. N. Sharpe, Jr., B. Yuan, R. Vaidyanathan, and R. L. Edwards, "Measurements of Young's Modulus, Poisson's Ratio, and Tensile Strength of Polysilicon”, $10^{\text {th }}$ Annual International Workshop on Micro Electro Mechanical Systems, Nagoya, Japan, 1/26-30/97, pp. 424-429. 